\title{
Synthetic post-AGB evolution
}

\author{
A. Frankowski ${ }^{\star}$ \\ Nicolaus Copernicus Astronomical Center, Polish Academy of Sciences, Rabiańska 8, 87-100 Toruń, Poland \\ Received 9 October 2002 / Accepted 13 May 2003

\begin{abstract}
A synthetic method for tracing the evolution of the post-AGB stars, based on existing numerical calculations, is presented. The main advantage of this procedure, based on the published numerical evolutionary sequences, is that it is simple, fast, and allows to treat post-AGB objects of arbitrary mass.
\end{abstract}

Key words. stars: evolution - stars: AGB and post-AGB

\section{Introduction}

Evolution of low- and intermediate-mass stars, considering their high abundance in stellar populations, is an important factor for the evolution of whole galaxies. Late stages of this evolution, associated with intense mass loss, are of particular interest, especially a transition from an inflated red giant of the Asymptiotic Giant Branch (AGB) to the hot compact core of a Planetary Nebula (PN). After climbing up to the tip of the Asymptotic Giant Brach and losing most of its hydrogen-rich envelope due to a copious wind $\left(10^{-5}-10^{-4} M_{\odot} / \mathrm{yr}\right)$, a star proceeds almost horizontally on the Hertzsprung-Russell diagram to the Planetary Nebula (PN) region. When the effective temperature reaches $\sim 3 \times 10^{4} \mathrm{~K}$, high energy photons emanating from the star ionize the previously ejected envelope - there appears a PN around the star and the Planetary Nebula Nucleus (PNN) stage begins.

A relatively short $\left(10^{3}-10^{4} \mathrm{yrs}\right)$ phase between AGB and PNN is called post-AGB or Proto-Planetary Nebula (PPN) phase. In this short time the stellar mass loss rate drops by several orders of magnitude. The wind gradually loses its dominant evolutionary role and is overcome by the thermonuclear reactions rate. The final mass of the star becomes fixed from now on - it will not change during later evolutionary phases. The structure of a post-AGB object is more or less onion-like. There is a compact $\mathrm{CO}$ core (with a radius of $\sim 0.01 R_{\odot}$ ) surrounded by a thin He layer with the H-burning shell on its top. (If the post-AGB phase coincides with the last thermal pulse, nuclear reactions take place at the bottom of the He layer, with the $\mathrm{H}$ shell being inactive). All this is in turn embedded within a diluted H-rich envelope (shrinking from $\sim 100 R_{\odot}$ at the end of AGB to $\sim 3 R_{\odot}$ at the beginning of the PNN phase). Then there is a stellar wind still emanating from the star and expanding shells of AGB matter even further from the center. The evolution of a post-AGB object is a sum of two processes: the evolution of the star, which is to become a Planetary Nebula

\footnotetext{
* e-mail: frankowski@ncac. torun.pl
}

Nucleus, and the evolution of an expanding proto-nebula, consisting of the matter expelled while on the AGB. The evolution of the star itself is rather independent of the proto-nebula state, but the reverse is not true: the development of the proto-nebula is significantly affected by the evolving central star characteristics. Hence, the evolution of the PPN's central star can be studied separately and then treated as an external input parameter for evolutionary calculations of the nebula. A reasonable description of the central star evolution is a necessary condition for a credible simulation of evolution of the entire post-AGB object.

The course of the post-AGB phase depends on the mass loss rate variations - how the wind depends on other stellar parameters and how rapidly it drops. However, the stellar wind evolution is hard to study (observationally - because the phase is short and hence objects are not very abundant, and theoretically - because the physical mechanisms driving the mass loss at AGB and post-AGB stages are only roughly known, and there is no consistent, credible model as yet). In the numerical models of the stellar evolution, the AGB to PNN transition is covered by semi-empirical analytical wind formulae, that are devised to produce mass loss rate just somewhere in-between the strong AGB wind and the much weaker PNN wind, with no firm calibration.

Not surprisingly, the post-AGB stage of stellar evolution is recently a subject of intense studies - both observational and theoretical. Since a pioneering work of Paczyński (1971), numerous studies on this topic have been presented (e.g. Härm \& Schwarzschild 1975; Schönberner 1979, 1981, 1983; Iben et al. 1983; Iben 1984; Kwok 1993; Vassiliadis \& Wood 1993, 1994; Blöcker 1995; Stanghellini \& Renzini 2000; Marigo et al. 2001). Various aspects of the post-AGB evolution are extensively discussed in Szczerba \& Górny (2001). For a general review on AGB, post-AGB and PNN phases the reader can consult Iben \& Renzini (1983) and Kwok (2000). Unfortunately, despite this effort, there exist only few detailed, time-dependent, numerical models of a star evolving through 
this transient phase between AGB and PN stages. There are two published sets of post-AGB data from full evolutionary calculations: one consists of results of Schönberner $(1981,1983)$ and Blöcker (1995) (hereafter BS) and second is from Vassiliadis $\&$ Wood (1993, 1994 hereafter VW) models. The VW results have been published for a more extensive grid of stellar parameters and are more reliable at the low-temperature region of late AGB phase (due to newer opacity tables used). However, their description of the mass loss rate evolution with stellar parameters seems to be less adequate. Abrupt switching from AGB wind to PNN wind in a quite arbitrarily choosen point causes the VW models to stay too long in the low effective temperature region (eg. Tylenda \& Stasińska 1994). A nice idea would be to mix somehow VW models with Blöcker's (or some other) wind description.

Both BS and VW models are calculated only for a very limited number of progenitor masses and for a specific mass loss description preferred by the authors. The main reason for this situation is the complexity of the problem and high computational cost of such modelling. But in some applications, e.g. in the population synthesis, it is essential to trace post-AGB evolution of many objects of different initial mass. This means that a fast, synthetic method of calculating the post-AGB evolution is needed.

Such methods in context of "post-AGB" evolution have been developed by Stanghellini \& Renzini (2000) and by Marigo et al. (2001), but both groups use the term "post-AGB" in a broad sense, i.e. for all stages following the AGB, and focus in fact on the PN phase. Accordingly, in both papers the modelling starts from the point where $\log T_{\text {eff }}=4$. "Post-AGB" defined as the transition phase from AGB to PN is not covered by their synthetic tracks.

Another method, focused mainly on this transition phase, is set forth in the present paper. The idea is to construct a synthetic relation between $\mathrm{H}$-rich envelope mass and effective temperature, parametrized by the $\mathrm{CO}$ core mass. This synthetic relation is calibrated by fitting analytical expressions to the grid of detailed numerical tracks. The main advantage of the proposed procedure is that it allows to treat post-AGB objects of arbitrary mass without excessive numerical costs. Preliminary results of this study have been presented in Frankowski (2003).

In Sect. 2, the general concept of the method along with its theoretical background is described. Putting VW evolutionary tracks into this scheme in Sect. 3 results in synthetic models of post-AGB stellar evolution for two metallicities (solar and SMC). Discussion and conclusions follow in Sect. 4.

\section{Theoretical background of the method}

Paczyński (1971) has shown that the evolution of a star transiting from a red giant to planetary nebula (PN) stage can be conveniently presented on a diagram displaying the hydrogen-rich envelope mass vs. the effective temperature. The $M_{\text {env }}-T_{\text {eff }}$ diagram has later been used to describe the post-AGB behavior by Schönberner $(1981,1983)$ and Blöcker (1995).

On such a diagram a star spends its AGB phase in the low temperature, high envelope mass region, with the effective temperature generally decreasing as the envelope mass decreases.

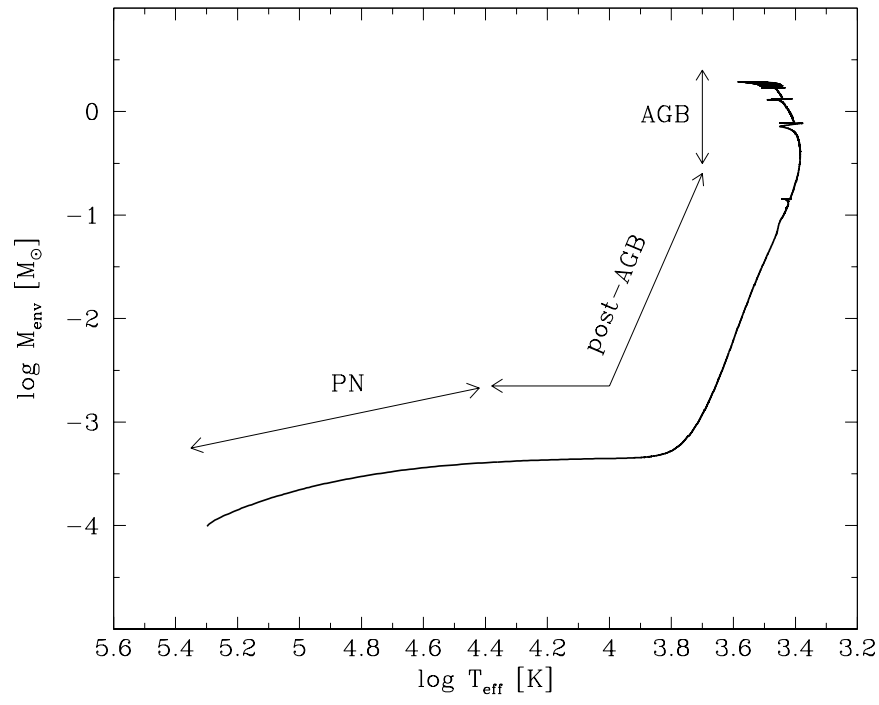

Fig. 1. The $M_{\text {env }}-T_{\text {eff }}$ relation for a Vassiliadis \& Wood's solar metallicity track of initial mass $M_{\text {ZAMS }}=2.5 M_{\odot}\left(M_{\text {core }}=0.668 M_{\odot}\right)$. AGB, post-AGB and PN phases are indicated. Spikes at low $T_{\text {eff }}$ result from He shell flashes.

The departure from the AGB begins when this trend is reversed. Then, the post-AGB phase shows up as a sharply downward and then a quasi-horizontal line, going from low to high effective temperatures with decreasing envelope mass. Some noise to this general tendency is introduced by He shell flashes (thermal pulses) - and if a He-flash occurs at or near the horizontal part of the relation, the picture is noticeably distorted. This case will not be considered in detail here. The simple method proposed in this paper is not suitable for modelling He-flash events, due to their non-equilibrium nature. In the available post-AGB evolutionary tracks there are also too few data to allow any systematical analysis of the stable He burning periods, that follow the flashes. Stable He burning does not usually last long and only one track in the VW dataset features stable He burning from AGB, through post-AGB and PN to the point where nuclear reactions cease. For these reasons, only H-burning post-AGB evolution can be considered within the proposed model.

At $T_{\text {eff }} \sim 3 \times 10^{4} \mathrm{~K}$ the stellar radiation starts to ionize the previously ejected matter and a PN is formed. When the nuclear reactions in the H-burning shell finally extinguish, the temperature stops rising and object enters the white dwarf cooling track. This last part is, however, beyond the scope of the present work. An example of a $M_{\text {env }}$ vs. $T_{\text {eff }}$ diagram resulting from detailed, numerical calculations of Vassiliadis \& Wood (1993, 1994) is shown in Fig. 1, together with arrows and labels indicating the evolutionary stages.

The post-AGB phase is relatively short $\left(\leq 10^{4} \mathrm{yrs}\right)$ and proceeds at nearly constant $\mathrm{CO}$ core mass (and, unless a shell flash occurs, also at almost constant luminosity). In this short time the mass of the hydrogen-rich envelope is reduced by orders of magnitude and the effective temperature increases dramatically. Hence, the $M_{\text {env }}$ vs. $T_{\text {eff }}$ relation can be treated - at least in the post-AGB phase - as characterized by a specific CO core mass, $M_{\text {core }}$. It is the core mass that determines the position 


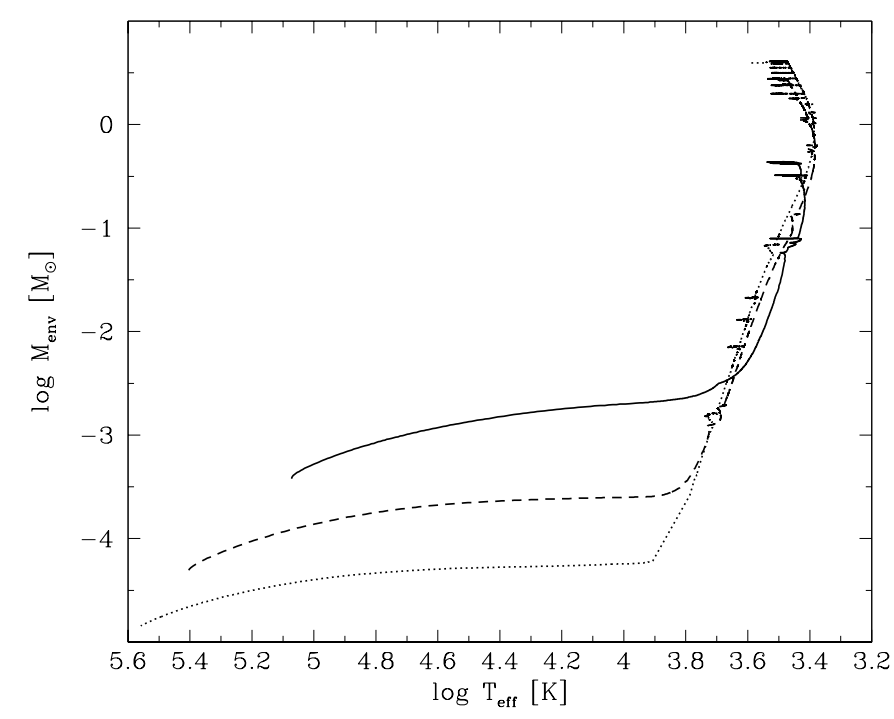

Fig. 2. $M_{\text {env }}-T_{\text {eff }}$ diagrams for solar metallicity VW tracks of ( $M_{\text {ZAMS }}$, $\left.M_{\text {core }}\right)=\left(1.0 M_{\odot}, 0.570 M_{\odot}\right)-$ solid line $\left(3.5 M_{\odot}, 0.755 M_{\odot}\right)-$ dashed line, and $\left(5 M_{\odot}, 0.900 M_{\odot}\right)$ - dotted line.

and shape of the stellar track on the $M_{\text {env }}$ vs. $T_{\text {eff }}$ diagram. $M_{\text {core }}$ governs also the stellar luminosity (Paczyński 1970; Uus 1970).

These facts were used to construct a $M_{\text {core-parametrized }}$ synthetic $M_{\mathrm{env}}-T_{\text {eff }}$ relation that allows to trace the evolution of a H-burning post-AGB object of arbitrary core mass. When accompanied with a mass loss rate formula and the $\mathrm{H}$ burning rate, this relation gives also the time evolution of the stellar parameters in this phase.

The procedure of constructing this synthetic relation was as follows. First, note that the general shape of the $M_{\text {env }}-T_{\text {eff }}$ relation is fairly similar for all the computed models, not only in the almost horizontal part, but also in the relatively cool, early phase of departure from the AGB stage, when $T_{\text {eff }}$ rises only slowly with decreasing envelope mass. Even stars suffering a late thermal pulse, before and after this event behave in a way very similar to the non flashing objects. This fact allows to use all the VW tracks in the constuction of the synthetic model (although the resulting synthetic evolution will not be able to reproduce late He-flashes). Main difference between the tracks is their position on the $M_{\text {env }}-T_{\text {eff }}$ plane. This is illustrated in Fig. 2, displaying $M_{\text {env }}-T_{\text {eff }}$ diagrams for solar metallicity $\mathrm{VW}$ tracks of $M_{\mathrm{ZAMS}}=1.0,3.5$, and $5 M_{\odot}$ (having, when on post-AGB, core masses $M_{\text {core }}=0.570,0.755$, and $0.900 M_{\odot}$, respectively). Otherwise the tracks are similar, but differ slightly in the curvature and extension of both the steep part and the quasi-horizontal one.

So, the general shape of the future synthetic $M_{\text {env }}-T_{\text {eff }}$ relation can be easily obtained by moving the curves corresponding to the numerical evolutionary tracks over the plane until they best cover each other and then making an average. As the shapes are noisy (due to the thermal pulses), differ in their span along both axes, and contain gaps (only H-burning periods should be considered), this fit has to be made by eye. When the curves match each other best, points along this bunch of lines can be choosen, that reflect its "average" course and therefore a general shape of the $M_{\text {env }}-T_{\text {eff relation. This general shape may }}$ be tabulated and it becomes a basis for the synthetic model. Then, moving this "average" curve over the plane to fit best actual $M_{\text {env }}-T_{\text {eff }}$ curves, one gets position coefficients of the synthetic diagram. Let us denote the coefficient governing the offset in the vertical direction (i.e. along $M_{\text {env }}$ axis) by $\Delta_{M}$, and the coefficient governing the offset in the horizontal direction (i.e. along $T_{\text {eff }}$ axis) by $\Delta_{T}$. It can be seen that these coefficients correlate with the CO core masses of given tracks. Next step is to account for shape differences between the computed models. It has been done by horizontal and vertical stretching of the "average" shape to fit the shape of a specific original model (while still keeping this synthetic curve in the right position on the $M_{\text {env }}-T_{\text {eff }}$ plane). This procedure gives a set of stretching coefficients in the vertical and horizontal direction $\left(\alpha_{M}\right.$ and $\alpha_{T}$, respectively), and also modifies values of the position coefficients mentioned above. Tests with VW and BS tracks have proved that inside a uniform grid of tracks all the four sets of coefficients show a good correlation with the $\mathrm{CO}$ core mass. Fitting analytical dependences on the $\mathrm{CO}$ core mass to all these coefficients leads to the final form of the synthetic $M_{\text {env }}-T_{\text {eff }}$ relation parametrized by $M_{\text {core }}$.

To get a synthetic $M_{\text {env }}-T_{\text {eff }}$ curve for a specific core mass one uses the aforementioned analytical expressions for stretching coefficients and for position coefficients (as functions of $\left.M_{\text {core }}\right)$ and applies them to the basic "average" tabulated relation. The diagram obtained in this way allows to determine the effective temperature for a post-AGB object characterized, for a given $M_{\text {core }}$, only by its envelope mass, $M_{\text {env }}$ (luminosity, $L$, can be provided from $M_{\text {core }}-L$ relation appropriate for a given set of original numerical models). For values of $M_{\text {env }}$ other than tabulated, linear interpolation between the two closest points can be used. Finally, supplying the nuclear burning rate and a wind formula translates this data into a time-dependent evolutionary sequence. A post-AGB object of core mass $M_{\text {core }}$ travels down its $M_{\text {env }}-T_{\text {eff }}$ relation at a rate determined by the nuclear burning and choosen expression for the mass loss rate.

\section{Results}

In principle, the above described procedure can be applied to any set of numerical evolutionary tracks that contain information required to construct $M_{\text {env }}-T_{\text {eff }}$ diagrams. The two aforementioned grids, BS and VW, differ in the parameter space coverage and in the opacities used. There is also some difference in "solar" abundance definition. Although it would be desired to use as much available data as possible, the incompatibility of these datasets proved to be too big and forced a decision to choose one of them. The VW grid has been choosen, because it consists of more models, is more uniform and uses more recent opacities. It also supplies more information on the earliest stages of an object's detachment from the AGB. Unfortunately, because of the difference in the opacity tables, a post-AGB evolution model based on the VW dataset will not reproduce the behaviour of the SB tracks.

The available VW grid consists of six tracks with metallicities $Z=0.016$ (solar), seven with $Z=0.008$ (LMC), seven 
Table 1. Points defining the average shape of the $M_{\mathrm{env}}-T_{\text {eff }}$ relation, used to construct the synthetic dependence for solar metallicity.

\begin{tabular}{cllll}
\hline \hline $\log M_{\text {env,av }}$ & $\log T_{\text {eff,av }}$ & & $\log M_{\text {env,av }}$ & $\log T_{\text {eff,av }}$ \\
\cline { 1 - 2 } \cline { 5 - 5 } 0.9 & 3.45 & & -2.6 & 3.707 \\
0.8 & 3.435 & & -2.85 & 3.75 \\
0.7 & 3.42 & & -3.0 & 3.79 \\
0.6 & 3.40 & & -3.1 & 3.83 \\
0.5 & 3.385 & & -3.12 & 3.85 \\
0.4 & 3.375 & & -3.14 & 3.90 \\
0.3 & 3.367 & & -3.15 & 4.00 \\
0.2 & 3.365 & & -3.16 & 4.15 \\
0.1 & 3.368 & & -3.18 & 4.33 \\
0.0 & 3.375 & & -3.2 & 4.46 \\
-0.2 & 3.395 & & -3.25 & 4.67 \\
-0.6 & 3.45 & & -3.3 & 4.80 \\
-1.0 & 3.51 & & -3.4 & 5.00 \\
-1.4 & 3.56 & & -3.6 & 5.26 \\
-1.8 & 3.605 & & -3.8 & 5.45 \\
-2.2 & 3.655 & & \\
\hline
\end{tabular}

with $Z=0.004$ (SMC), and two with $Z=0.001$. From these, only solar and SMC subgrids contain enough useful (i.e. not distorted too heavily by He-flashes) tracks to apply the procedure described in the previous section.

\subsection{Solar metallicity}

The solar metallicity subgrid consists of tracks computed for stars starting on ZAMS with masses $M_{\text {ZAMS }}=1.0,1.5,2.0$, 2.5, 3.5, and 5.0 $M_{\odot}$. The final core masses on the post-AGB are, respectively, $M_{\text {core }}=0.570,0.600,0.635,0.668,0.755$, and $0.900 M_{\odot}$. All these tracks have been used in the process of deriving the shape of the $M_{\text {env }}-T_{\text {eff }}$ relation and its modifying coefficients, as described in Sect. 2. The derived average shape $T_{\text {eff,av }}\left(M_{\text {env,av }}\right)$ of the $M_{\text {env }}-T_{\text {eff }}$ relation for solar metallicity is presented in Table 1. This average curve is closest to the $M_{\text {env }}-T_{\text {eff }}$ relation of the $M_{\text {core }}=0.755 M_{\odot}$ track.

To get a synthetic relation $T_{\text {eff }}\left(M_{\text {env }}\right)$ for a given $M_{\text {core }}$, the following expressions should be applied to each point from Table 1:

$\log M_{\text {env }}=\alpha_{M}\left(M_{\text {core }}\right) \times\left(\log M_{\text {env,av }}\right)+\Delta_{M}\left(M_{\text {core }}\right)$

$\log T_{\text {eff }}=\alpha_{T}\left(M_{\text {core }}\right) \times\left(\log T_{\text {eff,av }}\right)+\Delta_{T}\left(M_{\text {core }}\right)$.

Coefficients $\Delta_{M}\left(M_{\text {core }}\right), \Delta_{T}\left(M_{\text {core }}\right), \alpha_{M}\left(M_{\text {core }}\right)$, and $\alpha_{T}\left(M_{\text {core }}\right)$, used to positon and stretch the synthetic curve can be obtained from the following formulae, devised to produce - when the appropriate core mass, $M_{\text {core }}$, is inserted - lines fitting the results of Vassiliadis \& Wood's numerical calculations :

$$
\begin{aligned}
& \Delta_{M}= \begin{cases}3.011 M_{\text {core }}-2.559, & M_{\text {core }} \leq 0.658 \\
1.080 M_{\text {core }}-1.288, & M_{\text {core }}>0.658\end{cases} \\
& \Delta_{T}= \begin{cases}-12.204 M_{\text {core }}+8.267, & M_{\text {core }} \leq 0.646 \\
-3.185 M_{\text {core }}+2.439, & M_{\text {core }}>0.646\end{cases}
\end{aligned}
$$

Table 2. Same as Table 1, but for SMC metallicity.

\begin{tabular}{cllll}
\hline \hline $\log M_{\text {env,av }}$ & $\log T_{\text {eff,av }}$ & & $\log M_{\text {env,av }}$ & $\log T_{\text {eff,av }}$ \\
\cline { 1 - 2 } \cline { 5 - 5 } 0.9 & 3.46 & & -2.6 & 3.704 \\
0.8 & 3.44 & & -2.85 & 3.75 \\
0.7 & 3.425 & & -3.00 & 3.80 \\
0.6 & 3.41 & & -3.06 & 3.83 \\
0.5 & 3.4 & & -3.09 & 3.85 \\
0.4 & 3.395 & & -3.12 & 3.90 \\
0.3 & 3.391 & & -3.16 & 4.00 \\
0.2 & 3.39 & & -3.18 & 4.15 \\
0.1 & 3.392 & & -3.19 & 4.33 \\
0.0 & 3.397 & & -3.20 & 4.46 \\
-0.2 & 3.415 & & -3.24 & 4.67 \\
-0.6 & 3.455 & & -3.27 & 4.80 \\
-1.0 & 3.505 & & -3.34 & 5.00 \\
-1.4 & 3.55 & & -3.46 & 5.26 \\
-1.8 & 3.598 & & -3.58 & 5.45 \\
-2.2 & 3.645 & -3.78 & 5.70 \\
\hline
\end{tabular}

Table 1 together with Eqs. (1)-(5) constitute a synthetic model of post-AGB evolution for solar metallicity stars.

\subsection{SMC metallicity}

The SMC metallicity subgrid consists of tracks computed for stars starting on ZAMS with masses $M_{\text {ZAMS }}=0.89,1.0,1.5$, 2.0, 2.5, 3.5, and 5.0 $M_{\odot}$. The final core masses on the postAGB are, respectively, $M_{\text {core }}=0.558,0.593,0.640,0.672$, $0.692,0.855$, and $0.943 M_{\odot}$. The track $M_{\text {ZAMS }}=2.0 M_{\odot}$, $M_{\text {core }}=0.672 M_{\odot}$ has been dropped from the analysis due to an inconveniently placed He-flash, all the other tracks have been used. Obtained average shape $T_{\text {eff,av }}\left(M_{\text {env,av }}\right)$ of the $M_{\text {env }}-T_{\text {eff }}$ relation for SMC metallicity is presented in Table 2. Main difference compared to the solar metallicity average shape appears in the quasi-horizontal part of the curve.

As for the solar metallicity case, to get an SMC synthetic relation $T_{\text {eff }}\left(M_{\text {env }}\right)$ for a given $M_{\text {core }}$, Eq. (1) should be applied to each point from Table 2 . Appropriate coefficients $\Delta_{M}\left(M_{\text {core }}\right)$, $\Delta_{T}\left(M_{\text {core }}\right), \alpha_{M}\left(M_{\text {core }}\right)$, and $\alpha_{T}\left(M_{\text {core }}\right)$, for SMC metallicity are given by the following formulae:

$\Delta_{M}=\left\{\begin{array}{l}3.450 M_{\text {core }}-2.889, M_{\text {core }} \leq 0.690 \\ 0.746 M_{\text {core }}-1.022, M_{\text {core }}>0.690\end{array}\right.$

$\Delta_{T}= \begin{cases}-12.443 M_{\text {core }}+9.086, & M_{\text {core }} \leq 0.667 \\ -1.557 M_{\text {core }}+1.825, & M_{\text {core }}>0.667\end{cases}$ 


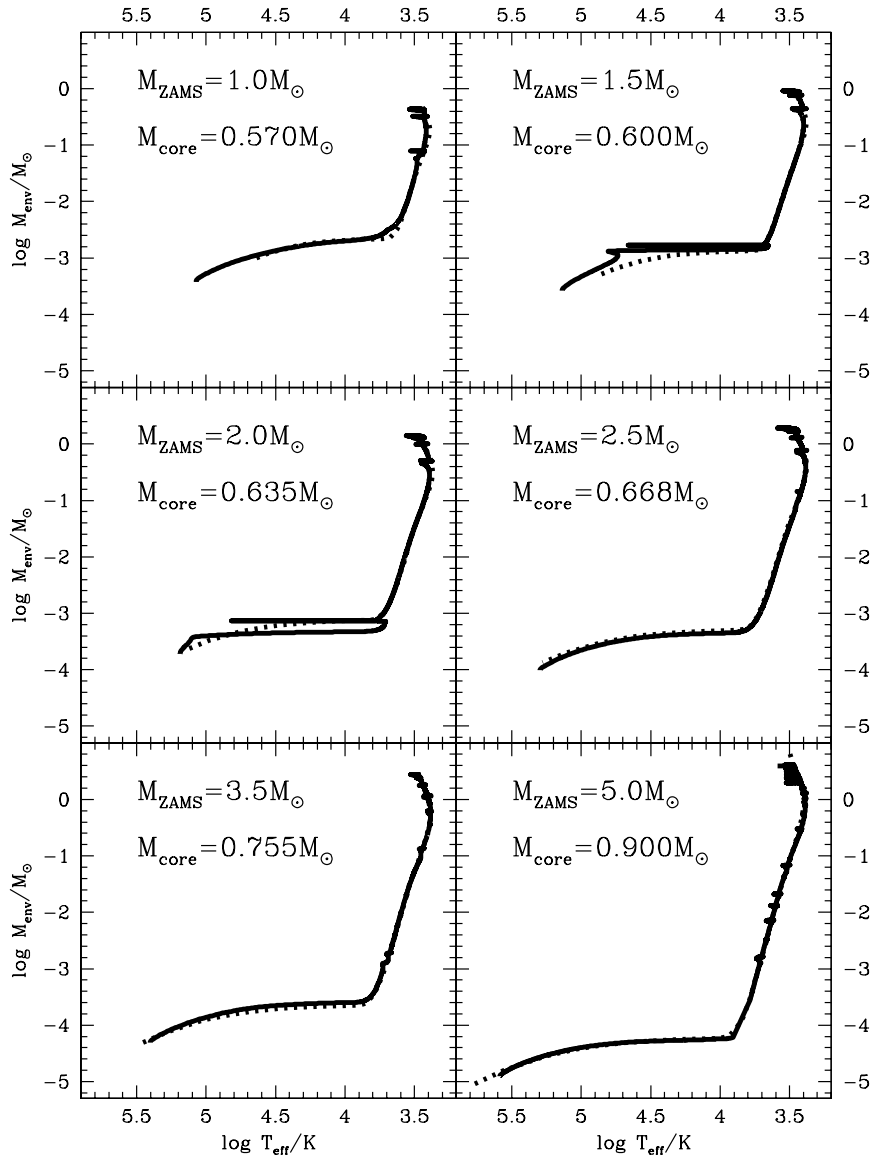

Fig. 3. Comparison of $M_{\text {env }}-T_{\text {eff }}$ diagrams for VW solar metallicity tracks (full lines) with the synthetic results (dotted lines). In each panel the core mass, $M_{\text {core }}$, of a given post-AGB object and initial mass of the respective VW track, $M_{\mathrm{ZAMS}}$, are indicated.

$\alpha_{M}=\left\{\begin{array}{l}3.186 M_{\text {core }}-1.287, M_{\text {core }} \leq 0.695 \\ 1.281 M_{\text {core }}+0.037, M_{\text {core }}>0.695\end{array}\right.$

$\alpha_{T}=\left\{\begin{array}{l}3.534 M_{\text {core }}-1.571, M_{\text {core }} \leq 0.668 \\ 0.504 M_{\text {core }}+0.452, M_{\text {core }}>0.668\end{array}\right.$

Table 2 together with Eqs. (1) and (6)-(9) constitute a synthetic model of post-AGB evolution for SMC metallicity stars.

\section{Discussion}

An obvious test for this model is a comparison of the model results with the original data it is based on. Such a comparison is given by Fig. 3 (solar metallicity case) and Fig. 4 (SMC metallicity) where the synthetic curves are overplotted on the original $M_{\text {env }}$ vs. $T_{\text {eff }}$ diagrams from numerical tracks of Vassiliadis $\&$ Wood. Panels in Figs. 3 and 4 show numerical and synthetic $M_{\text {env }}-T_{\text {eff }}$ diagrams for objects of different final mass, as indicated in each panel. Initial masses $M_{\text {ZAMS }}$ of respective VW tracks are also given. Full lines correspond to the numerical tracks, dotted - to the synthetic ones.

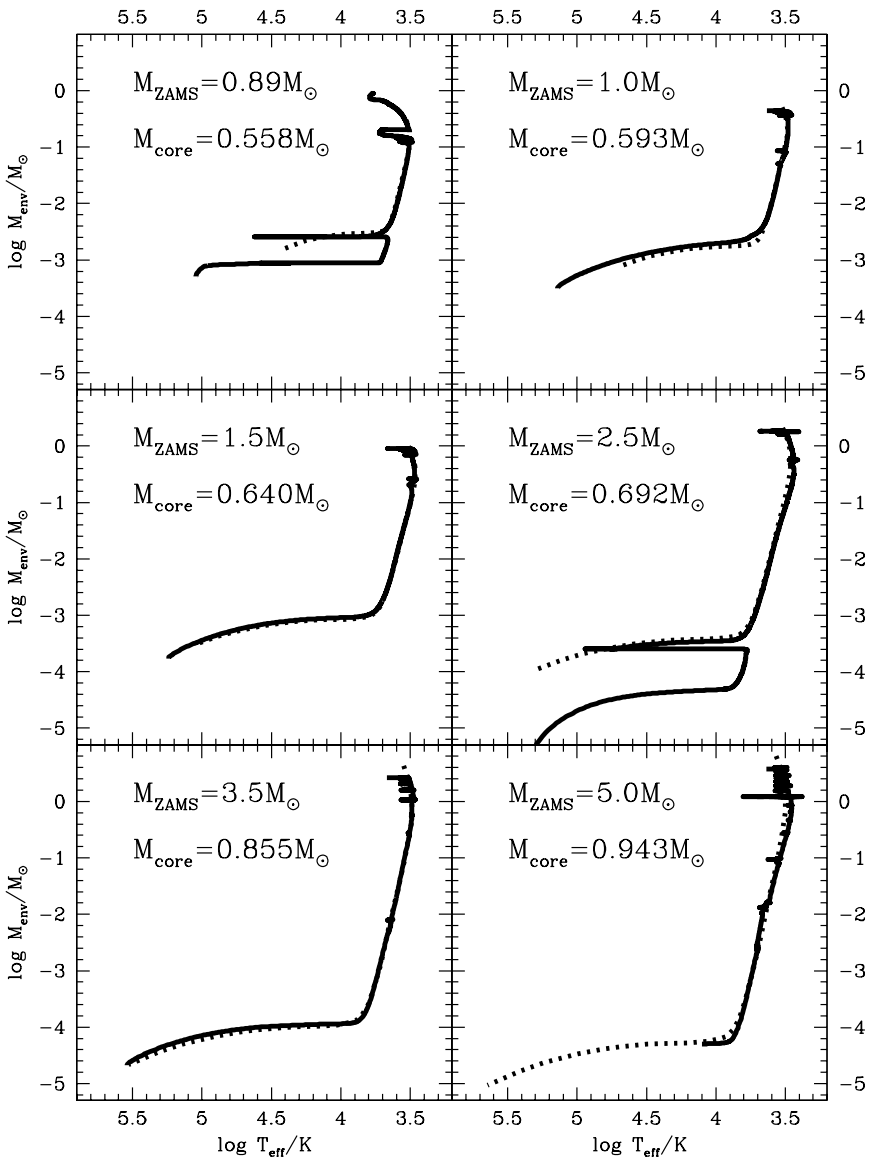

Fig. 4. Same as Fig. 3, but for SMC metallicity.

\subsection{Solar metallicity}

It can be seen that the overall match for solar metallicity models is good, with the exception of the least massive object of core mass $0.570 M_{\odot}$, which goes to much higher effective temperatures in the numerical calculations than is reached in the synthetic model. However, even in this case, at $\log T_{\text {eff }}<4.6$ the agreement is acceptable. Also tracks that exhibit a late He-flash $\left(M_{\text {ZAMS }}=1.5\right.$ and $\left.2.0 M_{\odot}\right)$ deviate from the synthetic relation during these flashes, but this is to be expected, as noted in Sect. 2.

Figure 5 compares the time evolution of the effective temperature in the original numerical tracks of solar metallicity and as reproduced by the proposed synthetic method. The mass loss prescription put into the synthetic calculations is the same as used by Vassiliadis \& Wood in their tracks. Only H-burning post-AGB tracks have been shown, but in other respects panels and symbols are as in Fig. 3. It can be seen that also here the agreement is satisfactory - the main timescales are reasonably reproduced. Again, the $M_{\text {core }}=0.570 M_{\odot}$ case exhibits the highest degree of deviation. A significant deceleration of the $T_{\text {eff }}$ evolution at $t \approx 4 \times 10^{4}$ yrs, which in the original VW track results from the AGB-to-PN wind regime switching, is not reproduced. This is because in the synthetic model, due to a more sharp bending of the synthetic $M_{\text {env }}-T_{\text {eff }}$ curve at $\log T_{\text {eff }} \approx 3.7$, compared to the original track (cf. upper left panel in Fig. 3) this regime switching occurs at lower envelope 


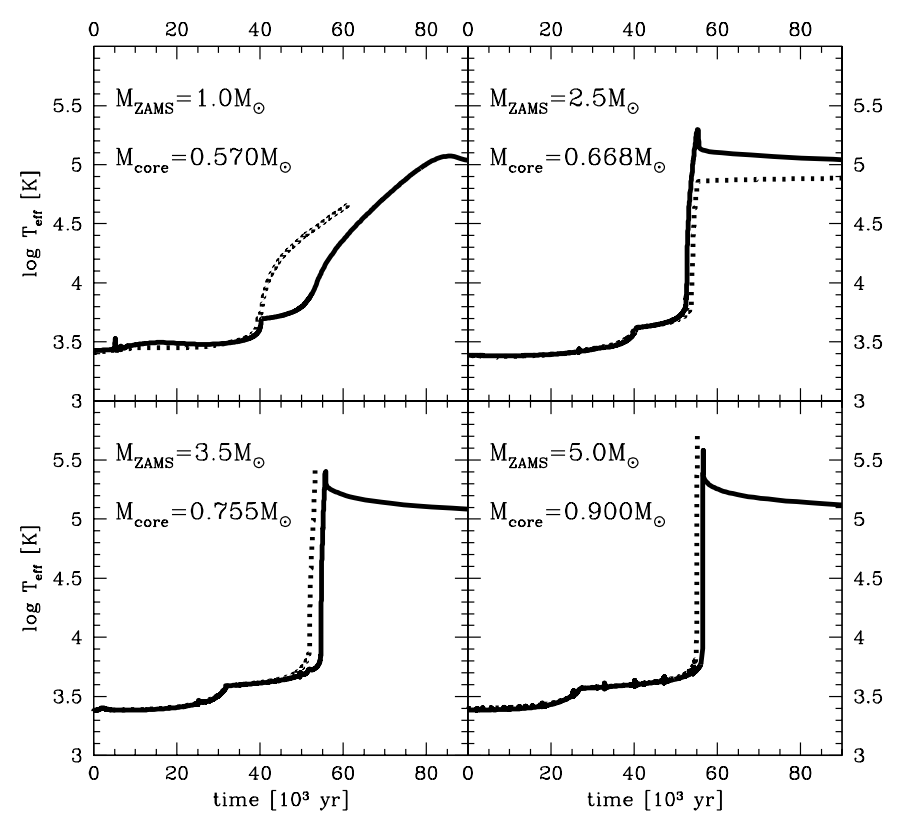

Fig. 5. The $T_{\text {eff }}$ time evolution of solar metallicity post-AGB objects resulting from the synthetic method (dotted lines), as compared to the H-burning VW tracks (full lines). Time, in units of $10^{3} \mathrm{yrs}$, starts at the AGB temperature minimum. Panels are labeled with object masses as in Fig. 3.

mass, already at the quasi-horizontal part of the $M_{\text {env }}-T_{\text {eff }}$ relation. At this point even minute changes in $M_{\text {env }}$ lead to large differences in $T_{\text {eff }}$ and this is why a weak PN-type wind suffices for fast $T_{\text {eff }}$ evolution. A close inspection of the synthetic $M_{\text {core }}=0.570 M_{\odot}$ curve in the upper left panel of Fig. 5 reveals that the $T_{\text {eff }}$ evolution does slow down for a while as the wind intensity drops, but this effect is much less pronounced than it is in the original track, where the wind regime change occurs at higher $M_{\text {env }}$, just on the bending before the quasi-horizontal part of the $M_{\text {env }}-T_{\text {eff }}$ curve. In other words, in the original track the PN-type wind starts at higher envelope mass and therefore has to strip off more matter before the evolution can accelerate again. Note, that although the $M_{\text {core }}=0.600 M_{\odot}$ and $0.635 M_{\odot}$ tracks experience a post-AGB He-flash, their behaviour near this characteristic bending in the $M_{\text {env }}-T_{\text {eff }}$ plane (at the beginning of the quasi-horizontal part of the diagram) is better reproduced by the synthetic model than in the $0.570 M_{\odot}$ case. This means that the model would reasonably represent time evolution of H-burning objects of $M_{\text {core }} \geq 0.6 M_{\odot}$. A conservative upper limit for using this method would be $M_{\text {core }}=0.9 M_{\odot}$, as this is the highest mass used in constructing the model. However, good agreement of the original and synthetic results at the high-mass end of the VW grid suggests that the applicability of the synthetic model can be assumed to extend at least to $M_{\text {core }} \approx 1 M_{\odot}$.

\subsection{SMC metallicity}

Comparison of $M_{\text {env }}-T_{\text {eff }}$ diagrams for SMC metallicity also reveals satisfactory agreement between numerical and synthetic results (Fig. 4), although the match is slightly less exact than in the solar metallicity case. Again, the least massive

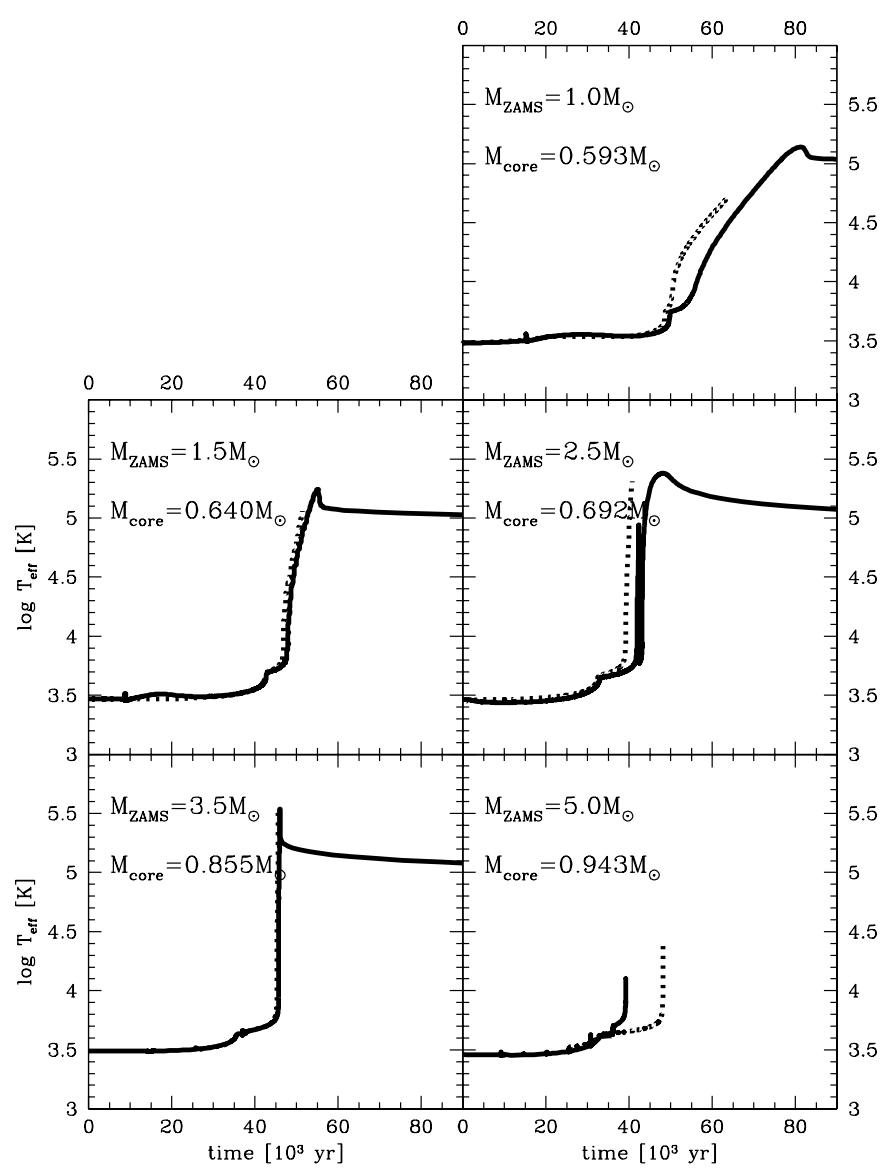

Fig. 6. Same as Fig. 5, but for SMC metallicity.

object $\left(M_{\text {core }}=0.558 M_{\odot}\right)$ breaks the picture, but now there is an additional reason - it is a late He-flash track. A late He-flash occurs also in the $M_{\text {core }}=0.692 M_{\odot} \mathrm{VW}$ track and the lower quasi-horizontal branch in this $M_{\mathrm{env}}-T_{\mathrm{eff}}$ diagram represents steady He burning that follows the flash. $\mathrm{H}$ burning does not recover in this case, as the flash occurs at extremely low envelope mass and this object ends it evolution as a He-burner. Obviously, the synthetic model should be compared to the upper branch, which corresponds to $\mathrm{H}$ burning - and in this part the match is good. Note also, that the numerical track for the most massive object, $M_{\text {core }}=0.943$, ends at $\log T_{\text {eff }} \approx 4.1-$ there is no data for higher temperatures in the publicly available VW track. In other cases, the agreement is acceptable at least up to $\log T_{\text {eff }} \approx 4.7$.

Figure 6 displays, for the SMC metallicity case, a comparison of the time evolution of the effective temperature in the VW tracks and as reproduced by the synthetic method (with VW mass loss prescription). As for solar metallicity, only H-burning post-AGB tracks are shown (in the case of the abovementioned $M_{\text {core }}=0.692 M_{\odot}$ object the original VW track is displayed only to the point of $\mathrm{H}$ burning cessation). The timescales are again reproduced quite well, but problems noted in solar abundance $M_{\text {core }}=0.570 M_{\odot}$ object are now present (to a lesser extent, though) for a higher mass star, $M_{\text {core }}=0.593$. Reasons are the same as for the least massive solar metallicity case. The most massive SMC metallicity object (bottom right panel in Fig. 6) also deserves a comment. 
There is a shift of $\sim 8.5 \times 10^{3} \mathrm{yrs}$ between the original VW and synthetic results for this case in the onset of the $T_{\text {eff }}$ evolution acceleration. This shift does not originate from an intrinsic inaccuracy of the proposed synthetic method, but is caused by a $\mathrm{He}$-flash occuring in the original VW track right after departure from the AGB. The flash causes a short ( 400 yrs) rejuvenation of a strong AGB wind, which at a very high rate (two orders of magnitude higher than before and after this episode) blows out a portion of the envelope and significantly shortens the postAGB phase following the He-flash. As no such event is possible in the synthetic model for H-burning post-AGB evolution, the post-AGB phase lasts longer in this case. Other objects are free of such difficulties and it seems that the applicability range of the synthetic method for SMC metallicity can be assumed similar to that of solar metallicity, i.e. $M_{\text {core }}=0.6-1 M_{\odot}$.

\subsection{Conclusion}

In summary, the conclusion is that the proposed synthetic evolution model is consistent with the numerical results it is based on. It can be used to obtain post-AGB evolutionary tracks of solar and SMC metallicity. Covered mass range is $M_{\text {core }}=0.57-0.90 M_{\odot}$ in the solar metallicity case and $M_{\text {core }}=$ $0.56-0.94 M_{\odot}$ for SMC metallicity, with possible extension to higher core masses in both cases. It should be stressed, that the model parameters obtained in this work do not reproduce the post-AGB evolution of Schönberner and Blöcker tracks, that are based on different opacity tables than these of $\mathrm{VW}$, as noted in Sect. 3.

The synthetic model of post-AGB stellar evolution presented here allows for fast computing of extensive grids of models for post-AGB objects of different parameters. It can be used e.g. as a part of synthetic stellar evolution or as input for hydrodynamical calculations of PPN and PN phases. Such applications are currently under development and their results will be published in next papers.
Acknowledgements. The author is very indebted to Prof. R. Tylenda for inspiration to work on this subject, his strong support during the project and careful reading of the manuscript. It is a pleasure to thank Prof. P. R. Wood for supplying numerical evolutionary tracks and to thank an anonymous referee for helpful suggestions that significantly improved the manuscript. This work has been supported from the grants No. 5.P03D.005.21 and No. 2.P03D.020.17 of the Polish State Committee for Scientific Research.

\section{References}

Blöcker, T. 1995, A\&A, 299, 755

Frankowski, A. 2003, in Planetary Nebulae: Their Evolution and Role in the Universe, ed. R. Sutherland, S. Kwok, \& M. Dopita, ASP Conf. Ser., in press

Härm, R., \& Schwarzschild, M. 1975, ApJ, 200, 324

Iben, I., Jr. 1984, ApJ, 277, 333

Iben, I., Jr., \& Renzini, A. 1983, ARA\&A, 21, 271

Iben, I., Jr., Kaler, J. B., Truran, J. W., \& Renzini, A. 1983, ApJ, 264, 605

Kwok, S. 1993, ARA\&A, 31, 63

Kwok, S. 2000, The Origin and Evolution of Planetary Nebulae, Cambridge Astrophys. Ser. 33 (Cambridge University Press)

Marigo, P., Girardi, L., Groenewegen, M. A. T., \& Weiss, A. 2001, A\&A, 378, 958

Paczyński, B. 1970, AcA, 20, 47

Paczyński, B. 1971, AcA, 21, 417

Schönberner, D. 1979, A\&A, 79, 108

Schönberner, D. 1981, A\&A, 103, 119

Schönberner, D. 1983, ApJ, 272, 708

Stanghellini, L., \& Renzini, A. 2000, ApJ, 542, 308

Szczerba, R., \& Górny, S. K., (ed.) 2001, Post-AGB Objects as a Phase of Stellar Evolution, ASSL 265 (Kluwer Academic Publishers)

Tylenda, R., \& Stasińska, G. 1994, A\&A, 288, 897

Uus, U. 1970, Nauch. Inf., 17, 32

Vassiliadis, E., \& Wood, P. R. 1993, ApJ, 413, 641

Vassiliadis, E., \& Wood, P. R. 1994, ApJS, 92, 125 